\title{
A Simple Equation Results in Decades of Technical Fun
}

\author{
Ronald C Lasky*
}

Dartmouth College, USA

*Corresponding author: Ronald C Lasky, Professor, Dartmouth College, Indium Corporation, USA.
Received Date: June 26, 2019

Published Date: June 28, 2019

\section{Opinion}

Decades ago, when I was getting my $\mathrm{PhD}$, I needed to calculate the density of a mixture of two polymers. I was tempted to use equation 1 below:

$$
\rho_{t}=x \rho_{1}+y \rho_{2} \text { equation } 1
$$

Where $\mathrm{x}$ is the mass fraction of polymer (or metal for a metal alloy), $y$ the mass fraction of polymer 2 , rho the respective densities and rho the total polymer or alloy density.

This equation seemed too obvious and I thought I should go to first principles and derive the correct equation. The result was equation 2 .

$$
\frac{1}{\rho_{t}}=\frac{x}{\rho_{1}}+\frac{y}{\rho_{2}} \text { equation } 2
$$

The derivation is in the appendix. Others have derived this equation before, as it is in some metallurgy texts.

Little did I know at the time that this equation would yield decades of "confrontation" and technical fun. Right after deriving this equation, some of my materials science grad student friends heard about it and asked for the equation. I suggested they derive it themselves and was a bit surprised that they had trouble doing it and sheepishly asked for my help in the derivation.

Years later, I began working in materials for electronic assembly, mostly solder alloys. About 15 years ago, I began blogging on topics in this materials field. I am still an active bloggeri today.

Shortly after beginning my blogging efforts, I posted on why equation 1 is incorrect and equation 2 is right. Thus, began over a

decade of queries to my blog about equation 2. An example follows (with names changed):Dr. Lasky,

My name is John Smith, a PhD metallurgist. I read with interest your article on calculating densities of alloys. Surely your formula:

$$
\frac{1}{\rho_{t}}=\frac{x}{\rho_{1}}+\frac{y}{\rho_{2}}
$$

can't be correct. I have been at ACME metals for over 30 years and have always used:

$$
\rho_{\text {total }}=x \times \rho_{x}+y \times \rho_{y}
$$

Please tell me it is not wrong!

Best, John Smith

Unfortunately, for Dr. Smith, he has been wrong for 30 years.

I then developed an Excel ${ }^{\circledR}$ spreadsheet ${ }^{\mathrm{ii}}$ to perform density calculations. I have had 100s of requests for the spreadsheet over the last decade.

As time went on, I was asked to verify the wet gold technique ${ }^{\mathrm{iii}}$. This technique is used to estimate gold content in jewelry scrap and gold ores. One thing that scares me is that it can only be used in a binary system (i.e. gold and one other metal or constituent.) I believe many users don't know this fact.

I continue to be asked to verify gold content formulas as a function of density and have incorporated much of this work into my classes at Dartmouth. I expect to have similar adventures in the future.

Appendix: Derivation of the Equation

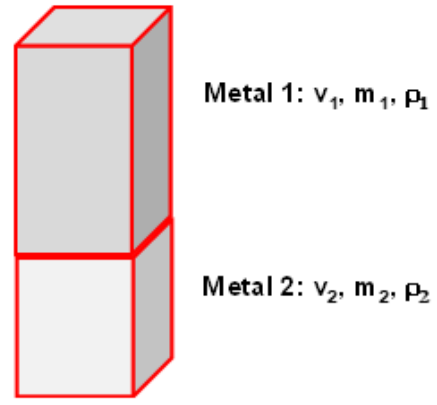


Assume the total volume upon mixing is constant.

$$
\begin{gathered}
v=\frac{m}{\rho} \\
v_{1}+v_{2}=v_{t} \rightarrow \frac{m_{1}}{\rho_{1}}+\frac{m_{2}}{\rho_{2}}+\frac{m_{t}}{\rho_{t}} \\
m_{1}+m_{2}=m_{t}, \quad x=\frac{m_{1}}{m_{t}}, \quad \mathrm{y}=\frac{m_{2}}{m_{t}}
\end{gathered}
$$

Dividing equation 1 by $m_{t}$,

$$
\frac{1}{m_{t}}\left(\frac{m_{1}}{\rho_{1}}+\frac{m_{2}}{\rho_{2}}\right)=\frac{1}{m_{t}} \frac{m_{t}}{\rho_{t}}
$$

$$
\frac{x}{\rho_{1}}+\frac{y}{\rho_{2}}=\frac{1}{\rho_{t}}
$$

\section{Acknowledgement}

None.

equation 1

\section{Conflict of Interest}

No conflict of interest.

\section{References}

1. https://www.indium.com/blog/dr-ron-lasky.php

2. Those interested in a copy send an email to rlasky@indium.com

3. https://www.indium.com/blog/wet-gold-a-technique-to-measuredensity-without-knowing-the-volume.php 